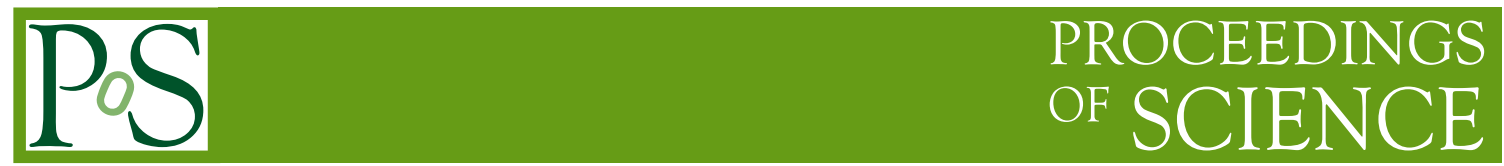

\title{
A fluid of diffusing particles and its cosmological behaviour
}

\author{
Zbigniew Haba* Institute of Theoretical Physics, University of Wroclaw, \\ Wroclaw,Poland \\ E-mail:zbigniew.haba@ift.uni.wroc.pl
}

\begin{abstract}
We discuss cosmological models with the rhs of Einstein equations determined by a sum of the energy-momentum of particles distributed over the phase space and a compensating cosmological term describing some other fields or matter. Then, a time depending cosmological term $\Lambda$ allows to preserve the energy-momentum conservation. We discuss a distinguished role played by the decay $\Lambda \simeq \frac{1}{t^{2}}$ and derive models experiencing such a behaviour.
\end{abstract}

Frontiers of Fundamental Physics 14

15-18 July 2014

Aix Marseille University (AMU) Saint-Charles Campus, Marseille, France

\footnotetext{
* Speaker.
} 


\section{Introduction}

We consider the metric

$$
d s^{2}=h_{\mu v} d x^{\mu} d x^{v}=d t^{2}-a(t)^{2}\left(\delta_{j k}+\gamma_{j k}\right) d x^{j} d x^{k}
$$

(in contradistinction to [1] $t$ denotes the cosmic time here; we restrict ourselves mostly to $\gamma=0$ ). Einstein equations have the form

$$
R^{\mu v}-\frac{1}{2} h^{\mu v} R=8 \pi G T^{\mu v},
$$

where $G$ is the Newton constant. The Einstein tensor on the lhs is covariantly conserved. Hence, $\left(T^{\mu v}\right)_{; \mu}=0$. We could insert on the rhs of eq.(1.2) the energy-momentum $T^{\mu v}$ of a collection of particles with initial conditions described by a probability distribution $\Omega$ on the phase space. If particle's dynamics is determined by classical evolution equations, then the conservation law is a consequence of the Liouville equation (where $\Gamma_{v \rho}^{\mu}$ are Christoffel symbols)

$$
\left(p^{\mu} \frac{\partial}{\partial x^{\mu}}-\Gamma_{v \rho}^{\mu} p^{v} p^{\rho} \frac{\partial}{\partial p^{\mu}}\right) \Omega=0
$$

when the energy-momentum tensor in eq.(1.2) is defined by

$$
\tilde{T}^{\mu v}=\sqrt{h} \int \frac{d \mathbf{p}}{(2 \pi)^{3}} \frac{1}{p_{0}} p^{\mu} p^{v} \Omega
$$

In eq.(1.4) $h$ is the determinant of the metric and $p_{0}$ is determined from the mass-shell condition $p_{\mu} p^{\mu}=m^{2}$ ( $m$ is the particle's mass). In eqs.(1.1)-(1.4) Greek indices run from 0 to 3, Latin indices denoting spatial components have the range from 1 to 3 . The deterministic approach (1.2)(1.4) must be modified if we describe only a part of the total system. In such a case we do not have the complete information. We must supplement our description by an extra term in the energymomentum

$$
T^{\mu v}=T_{D}^{\mu v}+\tilde{T}^{\mu v}
$$

where $T_{D}$ is the energy-momentum of a certain (dark) matter. From eq.(1.2) it follows

$$
\left(T_{D}^{\mu v}\right)_{; \mu}=-\left(\tilde{T}^{\mu v}\right)_{; \mu}
$$

\section{Diffusion and random dynamics}

It is well-known that classical dynamics in a random field can be approximated by diffusion. In [2] we have discussed relativistic dynamics in a random electromagnetic field $F$

$$
\begin{gathered}
m \frac{d x^{\mu}}{d \tau}=p^{\mu}, \\
m \frac{d p^{\mu}}{d \tau}=F^{\mu v} p_{v} .
\end{gathered}
$$

It follows from eqs.(2.1)-(2.2) that $\tau$ is the proper time and $p^{\mu} p_{\mu}=$ const. This is an essential requirement for relativistic dynamics. It is not simple to invent relativistic equations preserving the 
mass-shell. The geodesic equation could be treated as an example. However, in this case we do not know how to define a random metric. There is a simple example of random dynamics which applies to massless particles. We consider

$$
\begin{gathered}
\frac{d x^{\mu}}{d \tau}=p^{\mu}, \\
\frac{d p^{\mu}}{d \tau}=\phi(x) p^{\mu}+\sigma p^{\mu}+\lambda a^{2} p^{\mu} p^{v} u_{v}-\Gamma_{v \rho}^{\mu} p^{v} p^{\rho} .
\end{gathered}
$$

In eq.(2.4) we have introduced an observer velocity $u$ normalized as $h_{\mu v} u^{\mu} u^{v}=1$. From eq.(2.4)

$$
\frac{1}{2} \frac{d}{d \tau} p^{2}=\left(\phi(x)+\sigma+\lambda a^{2} u^{v} p_{v}\right) p^{2}
$$

Hence, if $p^{2}=0$ at $\tau=0$ then it remains zero forever. A function $\Omega(x(\tau), p(\tau))$ on the phase space satisfies the Liouville equation

$$
\partial_{\tau} \Omega=(X+Y) \Omega
$$

where

$$
X=p^{\mu} \frac{\partial}{\partial x^{\mu}}+p^{k}\left(\sigma+\lambda a^{2} p^{v} u_{v}\right) \frac{\partial}{\partial p^{k}}-\Gamma_{v \rho}^{k} p^{v} p^{\rho} \frac{\partial}{\partial p^{k}},
$$

and

$$
Y=p^{k} \phi(x) \frac{\partial}{\partial p^{k}}
$$

We have separated deterministic and random evolutions and imposed the initial condition $p^{2}=0$. We assume that $\phi$ is a random field with the covariance

$$
\langle\phi(x) \phi(y)\rangle=S(x-y)
$$

such that $S\left(x_{0}-y_{0}, \mathbf{x}-\mathbf{y}\right) \simeq \exp \left(-\tau_{c}^{-1}\left|x_{0}-y_{0}\right|\right)$ for a large time. Then, according to Kubo (see the discussion in [2]) the random motion can be approximated by the diffusion whose generator is defined by $\left\langle Y^{2}\right\rangle$ calculated for a small time (we have chosen $\sigma=2$ in eq.(2.4) in order to achieve a general coordinate invariance of eq.(2.10), see [3]). In the homogeneous metric ( $\gamma=0$ in eq.(1.1)) we obtain

$$
p^{\mu} \frac{\partial}{\partial x^{\mu}} \Omega=2 p^{k} p^{0} H \frac{\partial}{\partial p^{k}} \Omega+|\mathbf{p}| \frac{\partial}{\partial p^{k}} p^{k}|\mathbf{p}|^{-1}\left(\lambda a^{2} p^{v} u_{v}+\tau_{c} S(0) p^{j} \frac{\partial}{\partial p^{j}}\right) \Omega
$$

where $H=a^{-1} \partial_{t} a$ and $p^{0}=a|\mathbf{p}|$ (note that the diffusion equation in [1] was discussed mainly in conformal time). We denote $\beta=\lambda\left(\tau_{c} S(0)\right)^{-1}$. Then,

$$
\Omega_{E}=\exp \left(-a^{2} \beta u_{\mu} p^{\mu}\right)
$$

solves eq.(2.10). Hence, $\beta$ has an interpretation of the inverse temperature and $\kappa^{2}=\tau_{c} S(0)$ is the diffusion constant. We can get a solution of eq.(2.10) with an arbitrary initial condition which equilibrates to $\Omega_{E}$ (2.11) at $t=t_{0}$, starts at $t=t_{0}$ from the Jüttner equilibrium distribution (2.11) and subsequently continues as a solution of eq.(2.10) with $\lambda=\beta=0$ (describing a matter evolution without equilibration). Let

$$
A(t)=\int_{t_{0}}^{t} a(s) d s .
$$


Then, the above mentioned solution without an equilibration is [1]

$$
\Omega_{\theta}(t)=\theta^{3}(\theta+A)^{-3} \exp \left(-\kappa^{-2} \frac{a^{2}}{\theta+A}|\mathbf{p}|\right),
$$

where $\theta$ is a parameter which can be expressed by an equilibration temperature at $t=t_{0}$.

\section{Conservation laws}

The energy-momentum tensor (1.4) in the state (2.11) is conserved. We obtain from eqs.(1.2),(1.4) and (2.11) the standard Friedmann equation (ultrarelativistic case, flat space)

$$
\left(a^{-1} \frac{d a}{d t}\right)^{2}=\frac{8 \pi G}{3} \frac{1}{(2 \pi)^{3}} 24 \pi(\beta a)^{-4} .
$$

In general, the conservation law is

$$
\left(T^{\mu 0}\right)_{; \mu}=\partial_{t} T^{00}+3 a^{-1} \frac{d a}{d t} T^{00}+a^{-1} \frac{d a}{d t} \delta_{j k} T^{j k} .
$$

In a homogeneous universe we may write

$$
\tilde{T}^{\mu v}=\tilde{E} u^{\mu} u^{v}-\tilde{\pi}_{E}\left(h^{\mu v}-u^{\mu} u^{v}\right),
$$

where $\tilde{E}$ is the energy, $\tilde{\pi}_{E}$ the pressure and the four-velocity $u^{\mu}$ satisfies the condition

$$
h_{\mu v} u^{\mu} u^{v}=1 .
$$

For massless particles $\tilde{T}_{\mu}^{\mu}=0$. Hence,

$$
\tilde{\pi}_{E}=\frac{1}{3} \tilde{E}
$$

In general, we assume

$$
\tilde{\pi}_{E}=w \tilde{E} .
$$

For a general phase space distribution $\Omega$ the energy-momentum (1.4) is not conserved. We assume that the non-conservation comes from some other fields or matter which we describe by $T_{D}$ as in eq. (1.5). We represent the unknown energy $T_{D}$ in eq.(1.5) by a cosmological term $\Lambda$. Then

$$
T^{\mu v}=\tilde{T}^{\mu v}+h^{\mu v} \frac{\Lambda}{8 \pi G} .
$$

The energy conservation (1.6) (in the frame $u=(1, \mathbf{0})$ ) is expressed as

$$
-\partial_{t} \frac{\Lambda}{8 \pi G}=\partial_{t} \tilde{E}+3 a^{-1} \partial_{t} a\left(\tilde{E}+\tilde{\pi}_{E}\right)
$$

With the assumption (3.5) we have

$$
\left(\tilde{T}^{\mu 0}\right)_{; \mu}=\partial_{t} \tilde{E}+3 a^{-1} \partial_{t} a \tilde{E}(1+w) .
$$

Integration of eq.(3.7) gives (if $w$ is time-independent)

$$
\frac{\Lambda(t)}{8 \pi G}=\frac{\Lambda\left(t_{0}\right)}{8 \pi G}-\int_{t_{0}}^{t} a^{-3(1+w)} \partial_{r}\left(a^{3(1+w)} \tilde{T}^{00}\right) d r .
$$




\section{Decaying cosmological term}

It follows from eqs.(1.4),(3.6) and (3.9) that a model of the phase space distribution $\Omega$ determines $\Lambda$. As an example, the solution (2.12) gives

$$
\tilde{T}^{00}=\sqrt{h} \theta^{3}(\theta+A)^{-3} \int \frac{d \mathbf{p}}{(2 \pi)^{3}} \text { ap } \exp \left(-\kappa^{-2} \frac{a^{2}}{\theta+A} p\right)=\frac{1}{(2 \pi)^{3}} 24 \pi \kappa^{8} \theta^{3}(\theta+A) a^{-4} .
$$

From eq.(3.9) we obtain $\Lambda$. Then, Einstein equations (1.2) with the energy-momentum(4.1) and the cosmological term (3.9) read (for $w=\frac{1}{3}$ )

$$
\left(a^{-1} \frac{d a}{d t}\right)^{2}=\delta(A+\theta) a^{-4}-\delta \int_{t_{0}}^{t} d r a^{-3}+\frac{\Lambda}{3}\left(t_{0}\right),
$$

where

$$
\delta=\frac{1}{(2 \pi)^{3}} 48 G \pi^{2} \kappa^{8} \theta^{3} .
$$

We can find an explicit power-like solution of the integro-differential equation (4.2) by a fine tuning of parameters

$$
\begin{gathered}
a(t)=\delta^{\frac{1}{3}}(t-q), \\
\Lambda=8 \pi G \tilde{E}=\frac{3}{2}(t-q)^{-2}
\end{gathered}
$$

and $\left(t_{0}-q\right)^{2}=2 \theta \delta^{-\frac{1}{3}}$. Eq.(4.4) applies if $q<t_{0}$ because the integral in eq.(4.2) is divergent at $r=q$. The solution (4.4) defined on the interval $\left[t_{0}, \infty\right)$ does not achieve 0 reaching its minimum $a\left(t_{0}\right)=\delta^{\frac{1}{3}}\left(t_{0}-q\right)$. The solution (4.4) is interesting because it gives $H^{-1}$ (where $H$ is the present value of the Hubble constant) as the age of the universe in agreement with recent experimental data (see [4] for an explanation of a distinguished character of the linear evolution). The time evolution (4.5) of $\Lambda$ can also explain the present small value of the cosmological constant [5][6][7]. The $t^{-2}$ behaviour in $\Lambda \mathrm{CDM}$ model has been tested against observations in [6].

The result (4.5) is not surprising. Einstein equations (1.2) and eqs.(3.6)-(3.8) lead to the equation (for an arbitrary time-dependent $w$ )

$$
3 H^{2}+\frac{2}{(1+w)} \frac{d H}{d t}=\Lambda
$$

If $a=t^{\alpha}$ then $H=\alpha t^{-1}$ and

$$
\Lambda=\frac{1}{t^{2}}\left(3 \alpha^{2}-\frac{2 \alpha}{1+w}\right)
$$

We have got the $\Lambda$-term as an energy-momentum compensating correction for a particle system interacting with a random scalar field (2.4). We could consider a deterministic particle system interacting with a scalar field which has a Lagrangian of the form

$$
L=\frac{1}{2} h^{\mu v} \partial_{\mu} \phi \partial_{\nu} \phi-g \exp (-r \phi)
$$

Neglecting the particles in the first approximation the model of gravity plus the scalar field has the solution $\phi=\sigma \ln (t), a(t)=t^{\alpha}$ with $r \sigma=2$ and $\sigma(3 \alpha-1)=g r$; so that $\exp (-r \phi)=t^{-2}$ and (for a large $g$ ) $\alpha \simeq \sqrt{\frac{8 \pi G}{3}} \sqrt{g}[8]$. As a consequence, for a large $g$ we have $E \simeq-\pi_{E} \simeq g t^{-2}$. The pressure and the energy behave as if we had a cosmological term $\Lambda \simeq \frac{g}{t^{2}}$. 
As a next step we study the effect of diffusion and the decaying cosmological term upon the inhomogeneities of the metric $h_{\mu \nu}$. They have observational consequences on temperature fluctuations. We can look for a solution of the general diffusion equation [3] as a perturbation of the temperature

$$
\Omega=\exp \left(-a^{2}|\mathbf{p}|(\beta+\delta \beta)\right)
$$

We expand the temperature as a perturbation of the metric $\delta h_{\mu v}$. Thus far we have calculated only the tensor metric perturbations $\gamma_{j k}$ [9]. We have shown that the standard formulas for temperature fluctuations $\langle\delta \beta \delta \beta\rangle$ resulting from quantum metric fluctuations are modified by a damping factor $\exp \left(-\beta \kappa^{2} A(t)\right)$ implied by diffusion. The effect of diffusion on structure formation requires a solution of Einstein equations. This is now under investigation.

Interesting discussions with Andrzej Borowiec and Marek Szydlowski on the cosmological term are gratefully acknowledged.

The research is supported by Polish NCN grant DEC-2013/09/B/ST2/03455.

\section{References}

[1] Z. Haba, Class.Quant.Grav.31,075011(2014)

[2] Z. Haba,Journ.Phys.A46,155001(2013)

[3] Z. Haba, Class.Quant.Grav.27,095021(2010)

[4] F. Melia and A.S.H. Shevchuk,MNRAS, 419,2579(2012)

[5] J.L. Lopez and D.V. Nanopoulos, arXiv:hep-ph/9501293

[6] M. Szydlowski, arXiv:astro-ph/1502.04737

[7] J. Sola, arXiv:1402.7049

[8] F. Lucchin and S. Matarrese, Phys.Rev.D32,1316(1985)

[9] Z. Haba, Mod.Phys.Lett.A30, 1550036(2015) 